\title{
Advances in our understanding of the pathogenesis of celiac disease
}

\author{
Subrata Ghosh MD FRCPC FRCP FRCPE
}

C eliac disease is a common autoimmune disorder affecting approximately one in 100 individuals, and triggered by the ingestion of dietary gluten. Celiac disease has seen significant advances in the understanding of its pathogenesis. It is a model of an autoimmune disease with a defined environmental trigger. Celiac disease results from inappropriate $\mathrm{T}$ lymphocyte response to ingested gluten proteins in genetically susceptible individuals. Environmental factors incriminated include early life gluten exposure, short duration of breastfeeding, intestinal infections and changes in microbiota.

Recently, it has been shown in mice that retinoic acid acts as an adjuvant along with interleukin (IL)-15 to promote inflammatory responses to ingested antigen (1). Celiac disease is characterized by a T helper 1 (Th1) immune response, but unique gliadin-specific T helper 17 (Th17) lymphocytes have been recently described. These Th17 lymphocytes produce proinflammatory cytokines such as IL-17, interferon-gamma and IL-21, but also produce protective and regulatory cytokines such as IL-22 and transforming growth factor-beta (2). In addition FOXP3positive cells are significantly increased in the mucosa of celiac disease patients (3). Therefore, celiac disease is mediated through $T$ lymphocyte responses that deviate from normal homeostatic control.

The humoral component of the immune response - the autoantibodies - have been used as diagnostic tests including antibodies against gliadin, deamidated gliadin, endomysium and tissue transglutaminase. However, none of these tests are very useful in monitoring therapy, especially in the development of novel therapeutic agents. New tests are, therefore, required.

Ninety-five per cent of celiac disease patients carry the human leukocyte antigen (HLA) heterodimer DQ2, while the rest carry DQ8. Genome-wide association studies (4) continue to link celiac disease with non-HLA gene variants that control immune response. The aberrant immune response is mediated through antigen-presenting cells such as dendritic cells and T lymphocytes. In addition to celiac disease, there are gluten-sensitive individuals who develop symptoms on ingestion of gluten that are not associated with autoantibodies or other immune-mediated diseases. Many of these patients are on a self-imposed gluten-free diet, and the prevalence of celiac disease in such patients is $10 \%$, as diagnosed by the HLA-DQ2-gliadin tetramer test (5). Celiac disease is also associated with several autoimmune diseases with substantial genetic overlap.

It remains to be seen whether the understanding in pathogenesis leads to better approaches to prevention and treatment. Lifelong gluten-free diet intake is a significant commitment and often imperfect in patients. Several approaches are being investigated including eliminating the incriminating epitopes from alpha-gliadin, improving the intestinal barrier or reducing the immune response to gluten. Preventive strategies, early diagnosis and new approaches to treatment are likely to emerge as a result of better understanding of its pathogenesis.

Factors involved in the pathogenesis of celiac disease

1. Potential environmental triggers.

2. Amount of gluten intake and timing of introduction in diet during infancy.

3. Handling of gluten by tissue transglutaminase 2 .

4. HLA-DQ2 or HLA-DQ8: gene dose and gene type.

5. Immune gene polymorphisms that control T lymphocyte response to antigen presentation.

\section{REFERENCES}

1. DePaolo RW, Abadie V, Tang F, et al. Co-adjuvant effects of retinoic acid and IL-15 induce inflammatory immunity to dietary antigens. Nature 2011;471:220-4.

2. Fernandez S, Molina IJ, Romero P, et al. Characterization of gliadin-specific Th17 cells from the mucosa of celiac disease patients. Am J Gastroenterol 2011;106:528-38.

3. Brazowski E, Cohen S, Yaron A, Filip I, Eisenthal A.

FOXP3 expression in duodenal mucosa in pediatric patients with celiac disease. Pathobiology 2010;77:328-34.

4. Dubois PC, Trynka G, Franke L, et al. Multiple common variants for celiac disease influencing immune gene expression. Nat Genet 2010;42:295-302.

5. Brottveit M, Raki M, Bergseng E, et al. Assessing possible celiac disease by an HLA-DQ2-gliadin tetramer test. Am J Gastroenterol 2011 (Epub ahead of print).

\footnotetext{
${ }^{1}$ Department of Medicine, North Tower, Foothills Medical Centre, Calgary, Alberta

Correspondence: Dr Subrata Ghosh, Department of Medicine, Foothills Medical Centre, North Tower, 1403-29th Street Northwest, Calgary,

Alberta T2N 2T9. Telephone 403-944-8222, fax 403-944-1095, e-mail ghosh@ucalgary.ca

Received and accepted for publication March 18, 2011
} 


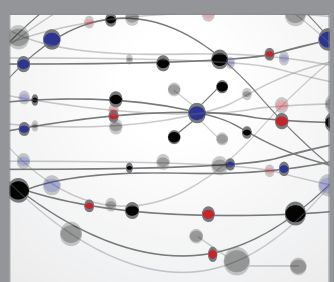

The Scientific World Journal
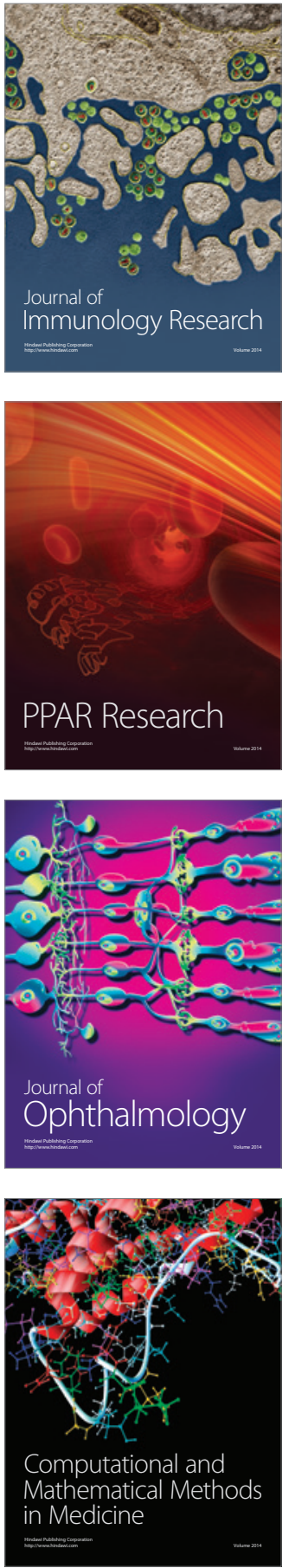

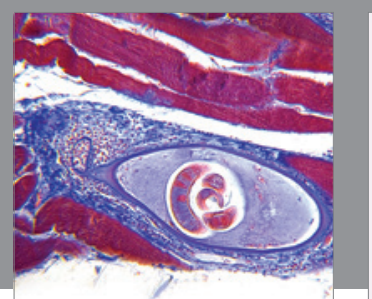

Gastroenterology Research and Practice

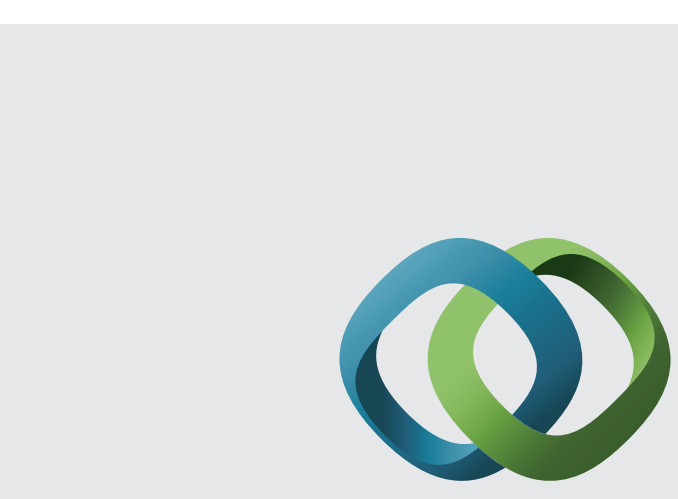

\section{Hindawi}

Submit your manuscripts at

http://www.hindawi.com
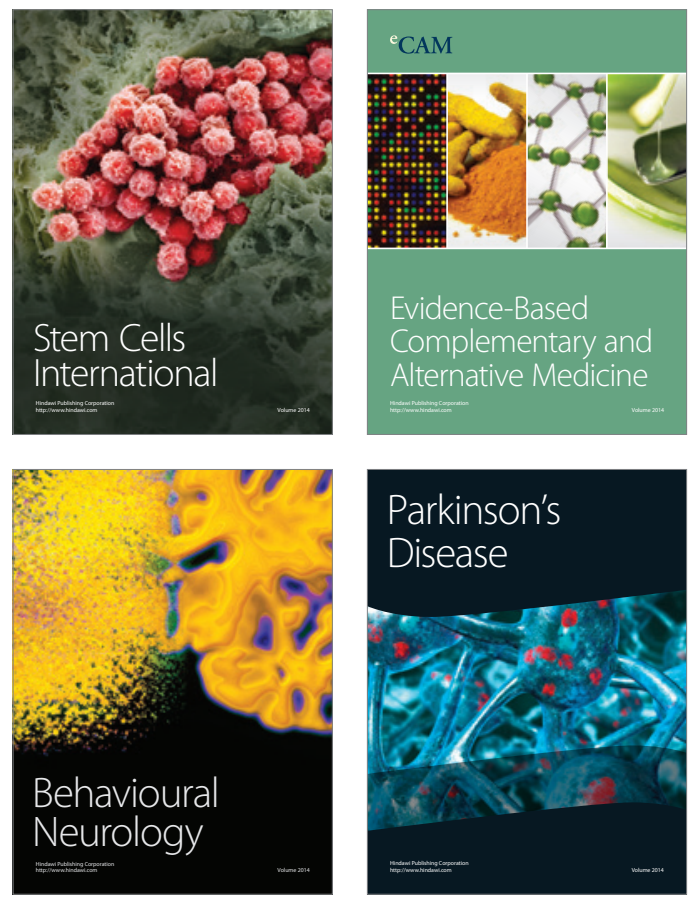
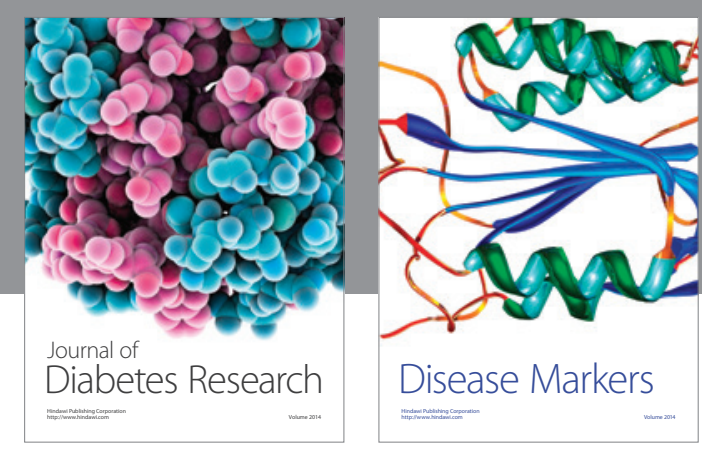

Disease Markers
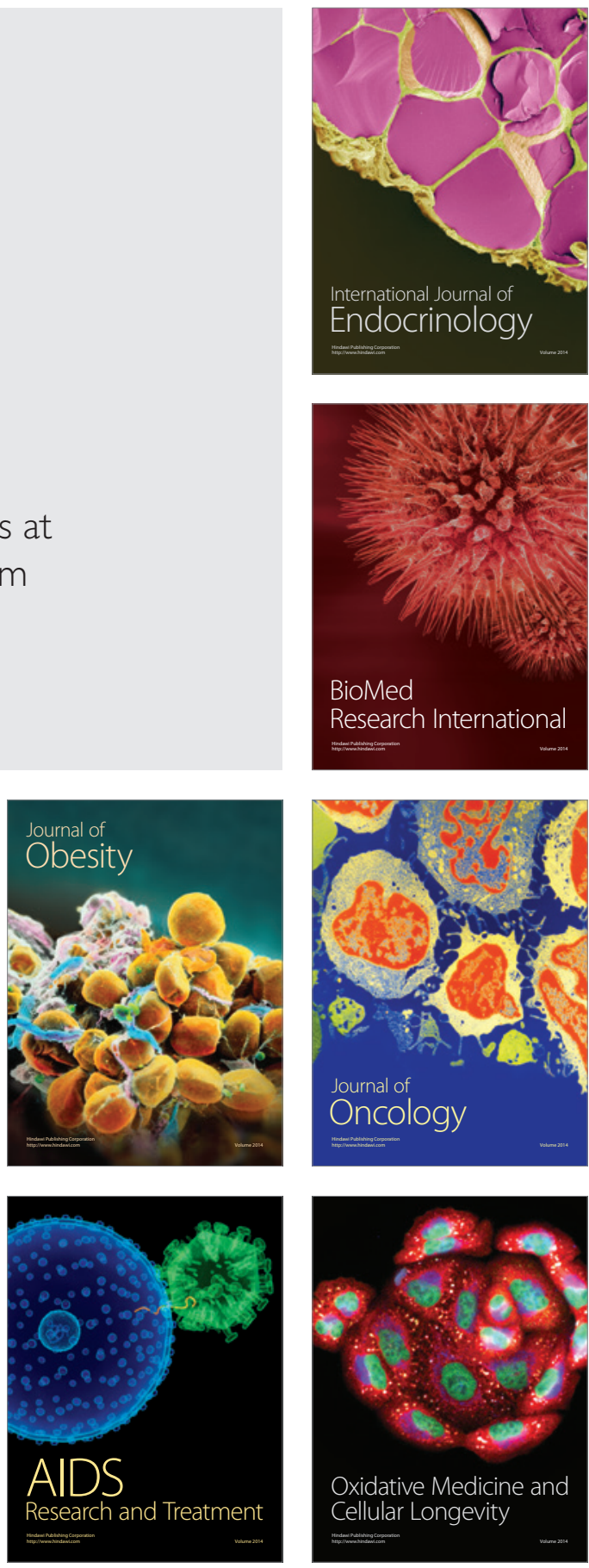